\title{
A study on the Dialogue Interpretation Based on Triangular Model
}

\author{
Hui Wang \\ School of Foreign Languages, Dalian Neusoft University of Information, Dalian, Liaoning, China \\ wanghui_yy@neusoft.edu.cn
}

Keywords: the interpretive theory; the triangular model; dialogue interpreting; fidelity

\begin{abstract}
Dialogue interpreting is a familiar form of interpreting that happened most frequently in our daily interpreted activities. In the dialogue interpreting, the two sides are maintaining a high level of interaction and are responsive to each other. In this thesis, the Triangular Model proposed by the interpretive theory is taken as the main theoretical frame of this thesis. The triangular model is used to analyze and judge the fidelity in dialogue interpreting in order to prove fidelity in dialogue interpreting, especially in the phase of deverbalization. And then, according to the three phases of the interpretive theory, three criteria are put forward to judge the fidelity in dialogue interpreting, namely, comprehending the speaker's intention, deverbalizing the source language, and reformulating in the target language.
\end{abstract}

\section{Introduction}

The trends of globalization cater to the needs of people coming together from all over the world to meet and communicate. With the rapid development of international exchanges, interpreting has gradually become a profession and played an increasingly important role in modern life. A word-toword interpretation can be easily done by those who are good at two languages. The difficult part lies in completing an interpreting task successfully. And fidelity is the most important principle for interpreters to abide by in interpreting. The importance of fidelity is the reason for this study. The main concern of this thesis is on the fidelity in dialogue interpreting based on the Seleskovitch's Triangular Model [1]. Practically, this research aims at explicating fidelity from the perspective of the Triangular Model and intends to provide interpreters with some criteria to improve the fidelity in their interpreting.

\section{The Frame of the Triangular Model}

\subsection{Comprehension}

As the first phase in the Triangular model, comprehension is considered as the basis of interpreting, which plays a vital role in comprehending the source language correctly and completely. It is known to all that the oral form will vanish when the speech is given by the speaker. And during this process, the interpreter has to understand and analyze the source language, and remember it for his or her further procession. Comprehension plays a very significant part in the process of interpreting, which serves as a solid foundation for the following phase. The interpreter needs to have high language capability and totally master two languages on the one hand. And on the other hand the interpreter should grasp the sense of the source language with the combination of the cognitive complements [2]. In this way, the interpreter can faithfully comprehend the communicative sense of the source language and lay a good foundation for the next step - deverbalization.

\subsection{Deverbalization}

The deverbalization phase is added between the comprehension and re-expression by the scholars of the Paris School [3], which is regarded as an innovation in the interpretive study. It seems that the phase of deverbalization is hard to get and comprehend and it seems invisible. However, it does exist and it is the core and foundation of the interpretive theory. Grasping this phase can facilitate the performance of interpreters. 


\subsection{Reformulation}

Re-expression is the last and the most obvious stage of interpreting procedure, which finally finish the communicative activity. The process of interpreting is a dynamic process of understanding, analyzing and reformulating thoughts. In this process, re-expression plays a role of reorganizing and reformulating the original content. As for the target language production, the interpreter should formulate their rendition on the basis of his or her earlier comprehending and analysis of the sense. Within this process, according to the basis of the deverbalization, the interpreter will formulate his or her target language production on the suggestive words or signs. The interpreter then adds, modifies and verifies the important grammatical constituents and finally makes a re-adjustment to the target language speech at the discourse level[4].

\section{The Application of the Triangular Model to the Judgment of Fidelity in Dialogue Interpreting}

\subsection{Comprehending the Speaker's Intention}

The nature of dialogue interpreting demands the interpreter participate in the process of the interpreting activity to better facilitate their communication and ultimately serve the purposes of the dialogue. The most important thing for the interpreters of dialogue interpreting is to smooth and spur the proceeding of communication and achieving the communicative aim of all the parties involved in the interaction. It is not the linguistic form, but the sense that the audience cares about and the interpreter should concentrate on [5]. If an interpreter limited oneself to the sentence pattern of the original speech without a complete understanding of sense, he or she may make his or her audience misunderstood and break communication. As for a faithful rendition, the implied meaning and intention of the speaker must be conveyed thoroughly and clearly.

\subsection{Deverbalizing the Source Language}

The phase "deverbalization" is really a necessary part in completing the task of communication. It is true that the process of deverbalization usually happened in the interpreter's mind, which cannot be easily observed by the audience though. It does not mean this phase does not exist. The reason is that what the audience heard is translated from the sense which is remembered by the interpreters in their brain, and the sense has already been deverbalized from the source language. It may be easier to illustrate this phase with the help of note-taking. As is known to all, human being's short-memory is limited. Therefore, many interpreters will use the skill of note-taking to help them finish their interpretation. If we have a chance to see the notes taken by a qualified interpreter, odds are that there are many symbols and few words, but parts of single words [6]. Unlike other kinds of interpreted events, the dialogue interpreting is a unique one with a high level of interaction. The interlocutors make their speech randomly which demands the interpreter pay full attention to the sense of the speaker, exerting the deverbalization to the advantage to facilitate their communication and ultimately serve the purposes of the dialogue. In this case, we could judge whether the interpreter has deverbalized the source language to evaluate the fidelity of the interpretation.

\subsection{Reformulating to the Target Audience in the Target Language}

Compared with the simultaneous interpretation, the interpreter in the dialogue interpreting enjoys more freedom in the reorganization and reformulation of his or her rendition on the original speech. The most important characteristic of dialogue interpreting is the face-to-face interaction. During the process of dialogue interpreting, the interpreter can observe the reaction of the audience to ensure whether the information is well received or not. If the audience seems not well understand what has heard, the interpreter should immediately make some further adjustments and explanations to make sure that the output is well received by the audience. To sum up, the interpreter has to reformulate his or her language into a final rendition which can be accepted and well understood by the target audience [7]. 


\section{Methods Derived from the Triangular Model}

\subsection{Logical Processing}

When people talk in Chinese, there are mainly short sentences with few conjunctions between them. While in English, it takes subject and predicate as the center of sentence, which contains a lot of complicated clauses. Compared with Chinese, English language has more relatives and conjunctions to imply the logical relationship.

The main idea of the interpretive theory is conveying the sense of the source language. During the process of comprehension, logic plays a crucial role, which should be delivered distinctly by the interpreter. Therefore, when translated Chinese into English, the interpreters need to catch the logical relationship between the sentences and make a logic processing to produce a long English sentence with relatives and conjunctions to stress the logic. Conjunction is an uninflected linguistic form that joins together sentences, clauses, phrases, or words, which includes coordinating conjunctions and subordinating conjunctions, such as: either...... or......, together with, while, however, as well as, since, so ...... that, unless and so on [8]. These conjunctions are essential in making English sentences, while they are seldom or even not employed in Chinese.

\subsection{Speech Gist Grasping}

The goal of deverbalization is to obtain the sense from the source language. In the dialogue interpreting, all the participants interact with each other frequently. In order to keep the conversations running smoothly without stop, the interpreter should try to extract and purify the sense of the original speech, which needs the interpreter to grasping the gist of speech. All these happen during the process of deverbalization. All the interpreters may encounter such circumstances that some of the original texts or utterances are not incoherent with each other. Sometimes the speech may not be precise or clear. While interpreting such sentences, a qualified interpreter should process the source speech and make some changes, such as omission or summary in order to make the speech understandable to the audience. He or she would try to analyze the logical structure beneath the original speech immediately, grasp the gist of it, and then give clear and complete interpretation to the audience. In dealing with the unrelated set of words or sentences, deverbalization plays a significant role in helping the interpreters to remember the sense of the utterance. The interpreter can use some skills to remember the gist of the source language rather than the verbal form of sentence, store the deverbalized input in his or her mind and finally reformulate them in the target language. Grasping the gist of speech is very important in dialogue interpreting and is one of the most frequently used methods.

\section{Summary}

The methods are derived from the Triangular Model to the interpreters to improve the fidelity in their interpreting. Through making efforts, the ideal interpreting methods derived from the triangular model have been analyzed with a number of examples, hoping to provide some guidance for the interpreters who are interested in dialogue interpreting to realize a faithful interpretation in their interpreting practice.

\section{References}

[1] Seleskovitch, D. Interpreting for International Conferences, translated by Stephanie Dailey and Norman Mcmillan. Washington: Pen and Booth, pp16-23,1998.

[2] Lederer, M. Translation, the Interpretive Model. Manchester: St. Jerome Publishing Ltd, pp 3445,2003.

[3] Seleskovitch, D. (1998). Interpretation, A Psychological Approach to Translating. New York: Gardner Press, pp1-10,1991. 
[4] Gang Bao.Interpretation Studies. Beijing: China Translation \& Publishing Corporation,pp 3742,2005 .

[5] Mason, I. Models and Methods in Dialogue interpreting Research. Beijing: Foreign Language Teaching and Research Press, pp 13-25, 2000.

[6] Baker, M. In Other Words---A Coursebook on Translation. Beijing: Beijing Foreign Language Teaching and Research Press,pp 31-35,2000.

[7] Heping Liu.Interpreting Skills: Approaches to Teaching Interpretation. Beijing : China Translation \& Publishing Corporation, PP108-109,2001.

[8] Merriam-Webster's collegiate dictionary. Springfield, MA: Werriam-Webster, 1993. 